John H. J. Wokke

\section{Fatigue is part of the burden of neuromuscular diseases}

Received: 24 September 2006

Accepted: 10 October 2006

Published online: 26 February 2007

Patients with neuromuscular diseases ask relatively simple questions. They want a timely diagnosis, and information about prognosis and effects of disease on their personal functional status. With respect to treatment, patients long for a cure but most understand that they will have to reconcile to rehabilitation or even palliative treatment.

With respect to diagnosis, neurologists are trained to detect impairments, neurological signs and symptoms such as motor weakness using the MRC scale, or a Babinski sign. Also, though usually not in a structured way neurologists try to evaluate disability, such as not being able to go up the stairs or cycling. Functional status may be measured with the sickness impact profile (SIP) or the modified Rankin scale. Finally effects of disease on participation, for example not being able to work or taking part in sports, and on perceived health will be estimated. Measures of disability, participation and perceived health are increasingly being used as out-

J. H. J. Wokke, MD ( $\square)$

Dept. of Neurology and Rudolf Magnus

Institute of Neuroscience

University Medical Center Utrecht

Rm G03. 228 P. O. Box 85500,

3508 GA Utrecht, The Netherlands

E-Mail j.wokke@umcutrecht.nl come measures in clinical trials [1].

Fatigue may be another underestimated symptom of neuromuscular disease [2]. That can be explained by ignorance as most patients with the exception of patients with myasthenia gravis or myotonic dystrophy do not spontaneously complain of fatigue. Many severely handicapped neuromuscular patients keep trying to participate in social life and work. In contrast, patients with the chronic fatigue syndrome have no or little objective signs and maximal decrease of participation and perceived health [2]. Yet, if fatigue would be a common additional feature of neuromuscular diseases, neurologists must be aware in order to better comprehend their patients and to develop new treatment strategies.

This issue of the Journal of Neurology contains two papers on fatigue in hereditary neuromuscular diseases. Dr. Hagemans did a cross-sectional study using a questionnaire with the Fatigue Severity Scale in 225 patients with late-onset Pompe disease [3]. About $67 \%$ had severe fatigue. Possible confounding factors were wheelchair dependency and respiratory support in about half of the patients, but $59 \%$ of patients without these aids also had severe fatigue. Previously this group investigated quality of life in lateonset Pompe patients using the SF36 [4]. Interestingly, they found that these patients score only slightly lower than the general population on the mental health domains, making depression or decreased vitality as explanations for fatigue unlikely $[3,4]$. The second paper shows that fatigue and pain can be relevant symptoms in facioscapulohumeral muscular dystrophy (FSHD) [5]. Both groups suggest that fatigue is probably underreported in these slowly progressive neuromuscular diseases.

Some caution with the role of confounding factors seems justified. The question whether patients with late onset Pompe disease and no respiratory aid have respiratory insufficiency that explains fatigue, can only be answered by actually measuring pulmonary function. In FSHD impaired shoulder function that is difficult to quantify, will affect quality of life and could explain fatigue.

Nevertheless, the notion that fatigue may be part of the phenotype of neuromuscular disease is important. To my opinion, the wear and tear from having to live a life long with the consequences of a neuromuscular disease may be one explanation for fatigue. Severe fatigue will affect participation and perceived health. Neurologists must learn to measure fatigue during history taking. Neurologists can also develop interventions. For instance, it has recently been demonstrated that simple bicycle exercise training may help to decrease fatigue in patients following Guillain-Barré syndrome [6].

\section{References}

1. Molenaar DSM, Vermeulen M, De Visser M, De Haan R (1999) Impact of neurological signs and symptoms on functional status in patients with peripheral neuropathy. Neurology 52:151-156

2. Chaudhuri A, Behan PO (2004) Fatigue in neurological disorders. Lancet 363: 978-988

3. Hagemans MLC, Van Schie SPM, Janssens ACJW et al. (2006) Fatigue: an important feature of late-onset Pompe disease. J Neurol 253:

4. Hagemans MLC, Janssens ACJW, Winkel LPF, et al. (2004) Late-onset Pompe disease primarily affects quality of life in physical health domains. Neurology 63:1688-1692 
5. Van de Kooi EL, Kalkman JS, Lindeman E, et al. (2006) Effects of training and albuterol on pain and fatigue in facioscapulohumeral muscular dystgrophy. J Neurol 253:
6. Garrsen MPJ, Bussman JBJ, Schmitz PIM, et al. (2004) Physical training and fatigue, fitness, and quality of life in Guillain-Barré syndrome and CIDP. Neurology 63:2393-2395 